\title{
Construction and evaluation of prognostic models for esophageal cancer patients with distant and non-distant metastases: providing a reference process for clinical diagnosis and treatment
}

\author{
Mingxin Zhang ${ }^{1,2 \#}$, Manli Cui ${ }^{1 \#}$, Qianqian Zuo ${ }^{3 \#}$, Li Wang ${ }^{4}, J_{i a} W^{1}{ }^{1}$, Lin Zhu ${ }^{1}$, Rong Yan ${ }^{1}$, Ning Lu ${ }^{1}$, \\ Honglin Yan $^{1}$, Lingmin Zhang ${ }^{5}$
}

${ }^{1}$ Department of Gastroenterology, The First Affiliated Hospital of Xi'an Medical University, Xi'an, China; ${ }^{2}$ Department of Internal Medicine, The Second Clinical Medical College of Shaanxi University of Chinese Medicine, Xianyang, China; ${ }^{3}$ Department of Oncology Geriatrics, The First Affiliated Hospital of Xi'an Medical University, Xi'an, China; ${ }^{4}$ Department of Scientific Research, The Affiliated Hospital of Xi'an Medical University, Xi'an, China; ${ }^{5}$ Department of Anesthesiology, First Affiliated Hospital of Xi'an Jiaotong University, Xi'an, China

Contributions: (I) Conception and design: M Zhang, M Cui, N Lu; (II) Administrative support: M Zhang, Q Zuo, L Wang; (III) Provision of study materials or patients: H Yan, L Zhang; (IV) Collection and assembly of data: J Wang, L Zhu; (V) Data analysis and interpretation: R Yan; (VI) Manuscript writing: All authors; (VII) Final approval of manuscript: All authors.

\#These authors contributed equally to this work and are co-first authors.

Correspondence to: Dr. Ning Lu; Prof. Honglin Yan. Department of Gastroenterology, The First Affiliated Hospital of Xi'an Medical University, No. 48 Feng Hao West Road, Xi'an, 710077, China. Email: 409143567@qq.com; yanhonglin666@163.com. Prof. Lingmin Zhang. Department of Anesthesiology, First Affiliated Hospital, Xi'an Jiaotong University, No. 277 Yanta West Road, Xi'an 710061, China. Email: zlm711@163.com.

Background: Although the current treatment for esophageal cancer has great technological progress, the 5 -year survival rate of patients is not optimistic. About $70 \%$ of patients with esophageal cancer are at an advanced stage at first diagnosis. These patients are prone to distant metastasis, and the prognosis is poor. Therefore, understanding the risk factors for distant metastasis in patients with esophageal cancer, combined with the prognosis of the patient, can aid in choosing the optimal diagnosis and treatment plan. Ultimately, it will improve the patient's survival time and quality of life. This research aims to construct a model for the risk assessment of distant metastasis in patients with esophageal cancer and prognostic models for patients with distant and non-distant metastases.

Methods: The Surveillance Epidemiology and End Results (SEER) database was used to select patients with esophageal cancer from 2010 to 2015 . The optimal cutoff point was selected for the age and tumor size variables using $\mathrm{X}$-tile. The nomogram was constructed using $\mathrm{R}$ software (The $\mathrm{R}$ Foundation for Statistical Computing).

Results: Gender, grade, T stage, N stage, and tumor size were independent risk factors associated with distant metastasis in patients with esophageal cancer. The concordance index (C-index) of the nomogram prediction model for whether the patient will have distant metastasis was 0.609. Age, grade, $\mathrm{T}$ stage, $\mathrm{N}$ stage, and tumor size were independent risk factors affecting the prognosis without distant metastasis. The C-index of the nomogram prediction model for patients with distant metastases was 0.590 . Age and $\mathrm{T}$ stage were independent risk factors affecting the prognosis of patients with distant metastases. The C-index of the nomogram prediction model was 0.543 . The combination of radiotherapy, chemotherapy, and primary surgery yielded the best overall survival for both patients with distant metastases and patients with nondistant metastases.

Conclusions: A comprehensive assessment of the risk of distant metastasis in patients with esophageal cancer, combined with prognosis prediction, is necessary to provide patients with a reasonable treatment plan.

Keywords: Esophageal cancer; distant metastasis; nomogram 
Submitted Jun 04, 2021. Accepted for publication Aug 19, 2021.

doi: 10.21037/jgo-21-429

View this article at: https://dx.doi.org/10.21037/jgo-21-429

\section{Introduction}

Esophageal cancer is one of the most common malignant tumors worldwide, with the seventh highest incidence rate and the sixth highest mortality rate globally (1). The current treatment methods for esophageal cancer include surgery, radiotherapy, and chemotherapy. Although great technological progress has been made, the 5 -year survival rate of patients is not optimistic. Most clinically diagnosed and treated patients are already in the middle and advanced stages are the main reason. Studies show that fewer than $40 \%$ of patients with esophageal cancer undergoing radical treatment have a 5 -year survival rate of about $25-50 \%$ (2). In terms of treatment choice $20-30 \%$ of patients choose to receive neoadjuvant chemotherapy and neoadjuvant chemoradiotherapy, but the effect is not significant, and early recurrence remains an intractable clinical challenge (3). One option, esophagectomy, is traumatic, with a long postoperative recovery time, many complications, a high postoperative mortality rate, and an adverse impact on the quality of life of patients. In contrast, endoscopic mucosal resection (EMR) and endoscopic submucosal dissection (ESD) preserve the integrity of the esophagus, with little surgical trauma; however, lymph node dissection remains difficult. There thus remains a lack of standard treatment options for patients with esophageal cancer. At present, experts are focusing their response strategies on the early detection, diagnosis and early treatment of esophageal cancer, which is an important measure to improve the prognosis of patients. Screening for esophageal cancer has gone from a large-scale social group census to the current mode of combining key screening of high-risk groups with opportunistic individual screening, which has improved the accuracy of screening, screening rate and early cancer detection rate.

Tumor invasion and metastasis are the most important biological characteristics of malignant tumors and have considerable impact on the treatment efficacy and prognosis of patients with esophageal cancer (4). About $70 \%$ of patients with esophageal cancer are at an advanced stage at first diagnosis, and advanced esophageal cancer is prone to distant metastasis. The prognosis of metastatic esophageal cancer, especially in cases with multiple metastases, is very poor (5). Therefore, understanding the risk factors for distant metastasis in patients with esophageal cancer, combined with the prognosis of the patient, can aid in choosing the optimal diagnosis and treatment plan, and ultimately improve the patient's survival time and quality of life. Our study establishes a simple and easy-to-use predictive model for predicting distant metastasis and prognosis of patients with esophageal cancer. Using the Surveillance Epidemiology and End Results (SEER) patient database, the most beneficial treatment methods for patients in the future can help reduce the current high mortality rate and increase the quality of life.

We present the following article in accordance with the TRIPOD reporting checklist (available at https://dx.doi. org/10.21037/jgo-21-429).

\section{Methods}

\section{Patient data}

A total of 2,181 esophageal cancer patients with complete follow-up data from 2010 to 2015 in the SEER database were selected as the study subjects. All patients had only 1 primary tumor of esophageal cancer. The follow-up deadline was December 31, 2016. Clinical death was the endpoint of follow-up. The patients' gender, age, race, tumor grade, $\mathrm{T}$ stage, $\mathrm{N}$ stage, primary site, pathological type, tumor size, and other information were extracted for summary analysis. The tumor-node-metastasis (TNM) staging is based on the 7th edition of the American Joint Committee on Cancer (AJCC), and the histological type is based on the International Classification of Diseases for Oncology, third edition (ICD-O-3). The study was conducted in accordance with the Declaration of Helsinki (as revised in 2013).

\section{Statistical methods}

SPSS 19.0 software (IBM Corp., Armonk, NY, USA) was used for single factor $2 \times \mathrm{C}$ chi-square test, logistic regression analysis, and Cox regression analysis. For the factors with the expected value of $<1$, Fisher's exact probability test was used. The forest map was drawn by Stata 15.1 software (StataCorp., College Station, TX, USA). $\mathrm{R}$ version 3.6.2 software (The R Foundation for Statistical 

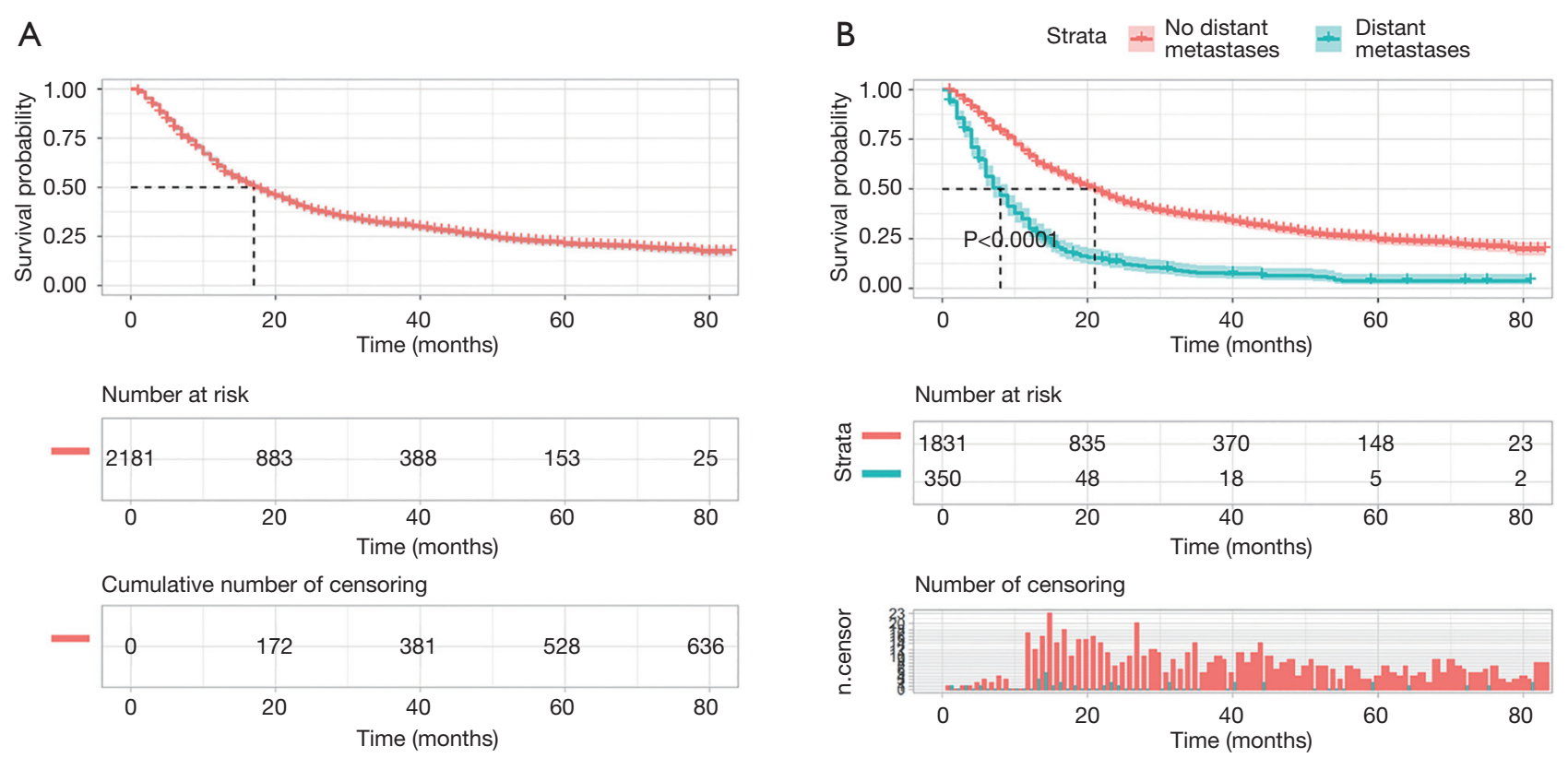

Figure 1 The survival curve for patients with esophageal cancer. (A) Overall survival curve for patients with esophageal cancer; (B) comparison of overall survival between patients with and without distant metastases.

Computing) was used to draw the survival curve and calculate the survival time and survival rate, and to build the predictive model. The receiver operator characteristics (ROC) curve was used to verify the constructed prediction model, which was completed using Medcalc medical statistical software (MedCalc Software Ltd., Ostend, Belgium).

\section{Results}

\section{Analysis of overall survival rate in patients with esophageal cancer}

The median survival time for patients of esophageal cancer was 17 [95\% confidence interval (CI), 15.71-18.30] months, and the 1-, 3-, and 5-year survival rates were $60.71 \%(95 \%$ CI: 58.61-62.81), 31.11\% (95\% CI: 28.96-33.21), and 20.92\% (95\% CI: 18.74-23.10), respectively (Figure 1A). Of the 2,181 patients, 350 patients had distant metastases, and 1831 patients had no distant metastases. The median survival time of patients with distant metastasis and those without distant metastasis was 8 (95\% CI: 6.95-9.05) and 21 (95\% CI: 19.55-22.46) months, respectively. The difference was statistically significant (logrank $\chi^{2}=263.74, \mathrm{P}<0.001$; Figure 1B).

\section{Selection of the best cutoff point}

$\mathrm{X}$-tile software was used to select the best cutoff points for the age and tumor size. The results showed that the best cutoff points for age were $\leq 72$ and $>72$ years, and the best cutoff points for tumor size were $\leq 63$ and $>63 \mathrm{~mm}$ (Figure 2).

\section{Analysis of related factors affecting distant metastasis and visualization of multivariate logistic regression in patients with esophageal cancer}

Univariate analysis results showed that gender, grade, $\mathrm{T}$ stage, $\mathrm{N}$ stage, and tumor size were the relevant risk factors for distant metastasis in patients with esophageal cancer (Table 1). Multivariate logistic regression analysis showed that gender, grade, $\mathrm{T}$ stage, $\mathrm{N}$ stage, and tumor size were independent risk factors for distant metastasis in patients with esophageal cancer (Table 1; Figure 3A).

A nomogram prediction model was constructed based on independent risk factors for distant metastasis (Figure 3B), with a concordance (C-index) of 0.609 (95\% CI 0.578 $0.640)$. The formula for risk was calculated as follows: Risk $=0.231 \times 10^{-2} \times$ total points $-1.677 \times 10^{-6} \times$ total points ${ }^{2}-$ $3 \times 10^{-9} \times$ total points ${ }^{3}+0.457$. The ROC curve was also used to verify the model, and the area under the curve (AUC) was 

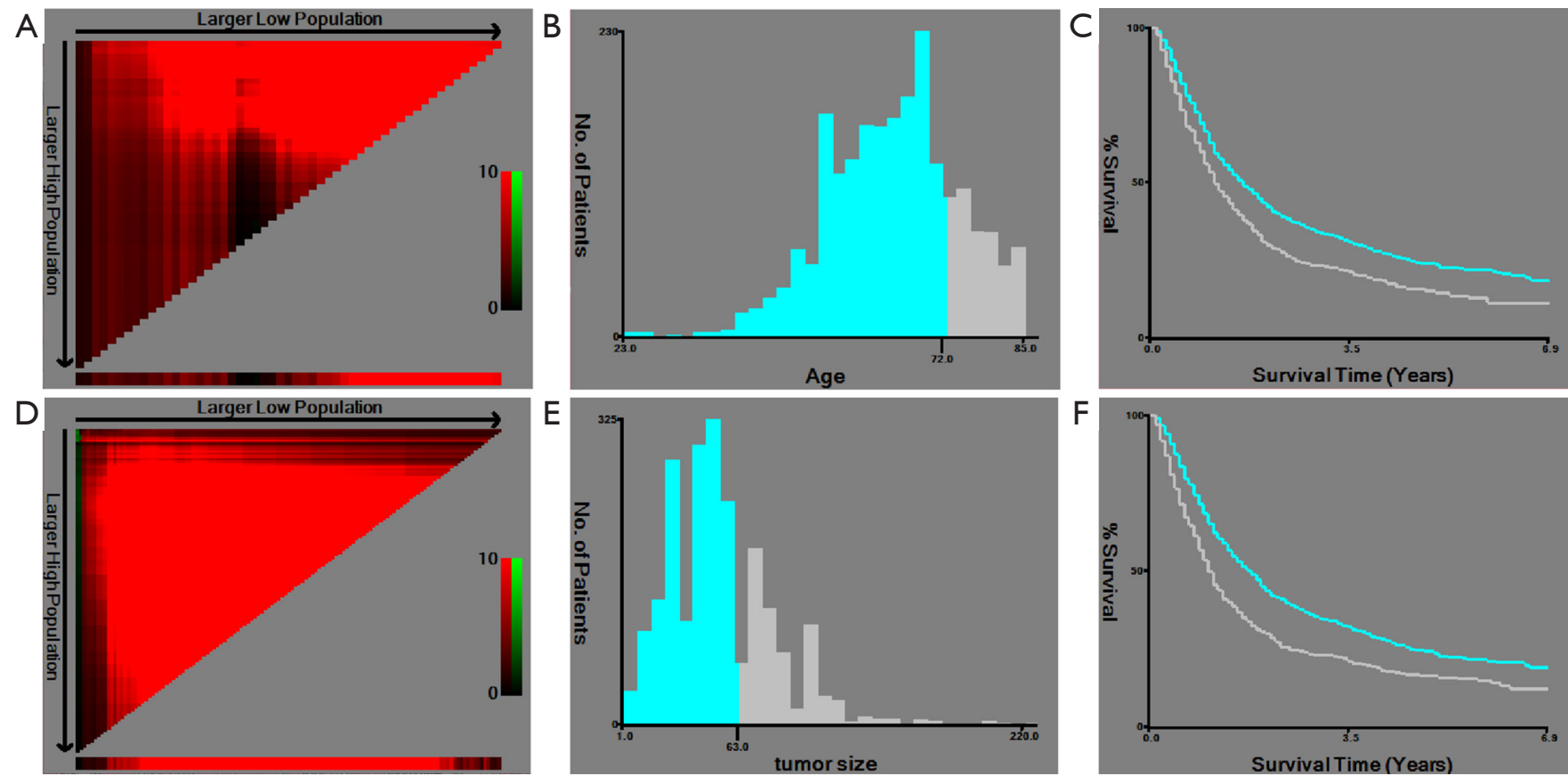

Figure 2 Selection of the best cutoff points. (A) The coloration of the plot represents the strength of the association, ranging from low (dark, black) to high (green or red). Indirect associations between age and survival are in red, whereas positive associations are in green; (B) the histogram shows the optimal cutoff point of age (blue: age $\leq 72$ years old, gray: age $>72$ years old); (C) the Kaplan-Meier curve shows the corresponding cutoff point of age (blue: age $\leq 72$ years old, gray: age $>72$ years old); (D) the coloration of the plot represents the strength of the association, ranging from low (dark, black) to high (green or red). Indirect associations between tumor size and survival are in red, whereas positive associations are colored green; (E) the histogram shows the optimal cutoff point of tumor size (blue: tumor size $\leq 63$ mm, gray: age $>63 \mathrm{~mm}$ ); (F) the Kaplan-Meier curve shows the corresponding cutoff point of tumor size (blue: tumor size $\leq 63$ mm, gray: age $>63 \mathrm{~mm}$ ).

Table 1 Univariate analysis and multivariate logistic regression analysis of distant metastasis in patients with esophageal cancer

\begin{tabular}{|c|c|c|c|c|c|c|c|c|}
\hline Variable & $\begin{array}{c}\text { Cases } \\
(n=2,181)\end{array}$ & \multicolumn{4}{|c|}{ Univariate analysis } & \multicolumn{3}{|c|}{ Multivariate Logistic regression analysis } \\
\hline Gender & & & & 6.759 & 0.009 & & 6.252 & 0.012 \\
\hline Male & 1,746 & 298 & 1,448 & & & 1 & & \\
\hline Age (years) & & & & 0.727 & 0.394 & & & \\
\hline$\leq 72$ & 1,688 & 277 & 1,411 & & & & & \\
\hline$>72$ & 493 & 73 & 420 & & & & & \\
\hline Race & & & & 6.553 & 0.083 & & & \\
\hline
\end{tabular}

Table 1 (continued) 
Table 1 (continued)

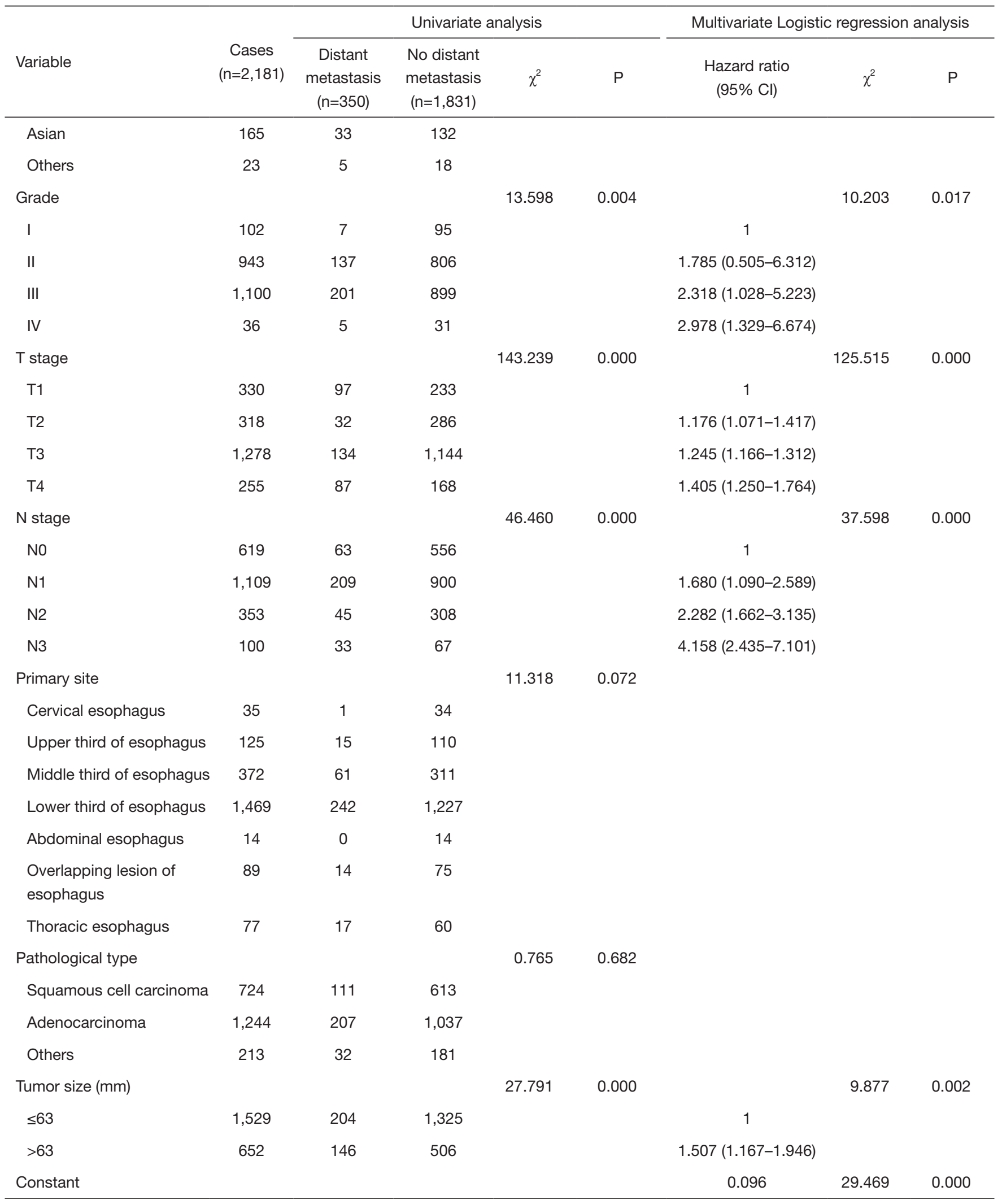


A

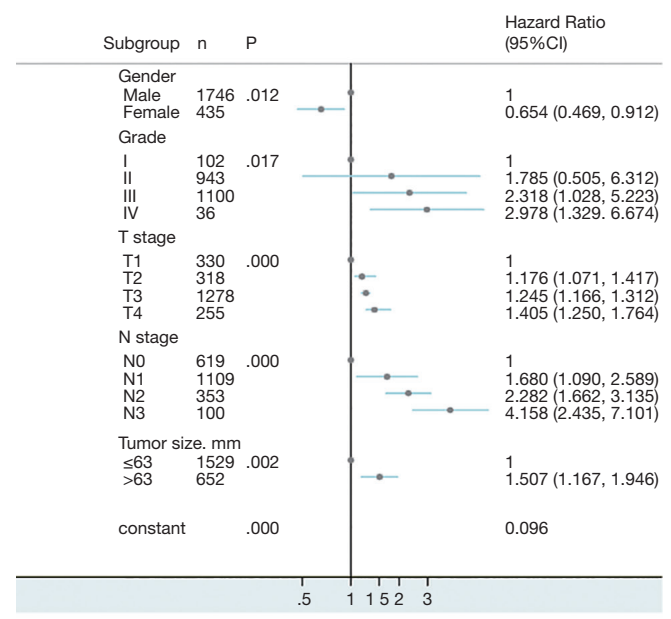

B



C

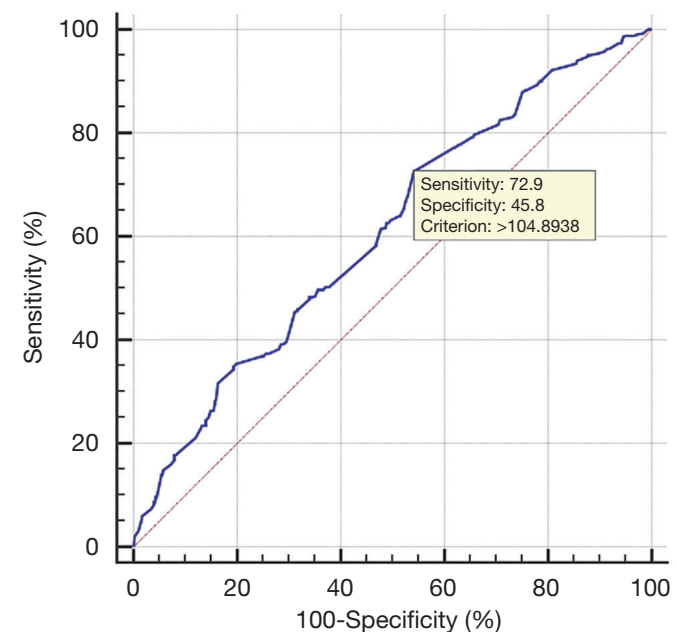

Figure 3 Multivariate logistic regression analysis and visualization of multivariate logistic regression in patients with esophageal cancer. (A) Forest plots for multivariate logistic regression analysis; (B) construction of a nomogram for risk of distant metastasis in esophageal cancer; (C) validation of a distant transfer risk prediction model based on ROC curve.
0.609 (95\% CI: 0.589-0.630; $\mathrm{z}$ statistic $=6.754 ; \mathrm{P} \leq 0.001$ ). In addition, the sensitivity, specificity, and criterion values were 72.9, 45.8, and 104.8938, respectively (Figure 3C).

\section{Prognostic analysis of patients without distant metastasis}

The results of univariate and multivariate Cox regression analysis showed that age, grade, $\mathrm{T}$ stage, $\mathrm{N}$ stage, and tumor size were independent risk factors affecting the prognosis of patients without distant metastasis (Table 2; Figure 4A,4B).

A nomogram prediction model was constructed based on independent risk factors affecting distant metastasis (Figure 4C), with a C-index of 0.590 (95\% CI: 0.571-0.609). The formulae for survival were calculated as follows: 1 -year survival $=3.867 \times 10^{-6} \times$ total points ${ }^{3}-0.407 \times 10^{-3} \times$ total points $^{2}-0.928 \times 10^{-2} \times$ total points $+0.809 ; 3$-year survival $=$ $1.323 \times 10^{-5} \times$ total points ${ }^{3}-0.367 \times 10^{-3} \times$ total points ${ }^{2}-0.019$ $\times$ total points +0.579 ; and 5 -year survival $=1.358 \times 10^{-5}$ $\times$ total points ${ }^{3}-0.168 \times 10^{-3} \times$ total points $^{2}-00.022 \times$ total points +0.470 . ROC curve was also used to verify the model, and the AUC was 0.627 (95\% CI: 0.605-0.649; $\mathrm{z}$ statistic $=9.339 ; \mathrm{P}<0.001)$. In addition, the sensitivity, specificity, and criterion values were 61.1, 58.9, and 9.0892, respectively (Figure 4D).

In all, 89 patients received radiotherapy only, 868 patients received radiotherapy + chemotherapy, and 844 patients received radiotherapy + chemotherapy + primary site surgery. No analysis was performed for other treatments due to the small number of patients in other categories. The results showed that the median survival time of patients receiving triple therapy was 39 (95\% CI: 33.669-44.331) months with the best prognosis, while the median survival time of patients receiving radiotherapy only was 7 (95\% CI: 4.867-9.133) months with the worst prognosis (Table 3; Figure 4E).

\section{Prognostic analysis of patients with distant metastases}

The results of univariate and multivariate Cox regression analysis show that age and $\mathrm{T}$ stage were independent risk factors affecting the prognosis of patients with distant metastases (Table 4, Figures 5A,5B).

A nomogram prediction model was constructed based on independent risk factors for distant metastasis (Figure 5C), with a C-index of 0.543 (95\% CI: $0.517-0.569)$. The formulae for survival were calculated as follows: 1-year survival $=0.341-0.013 \times$ total points; and 3 -year survival $=$ $0.101-0.692 \times 10^{-2} \times$ total points. The ROC curve was also 
Table 2 Univariate and multivariate Cox regression analysis

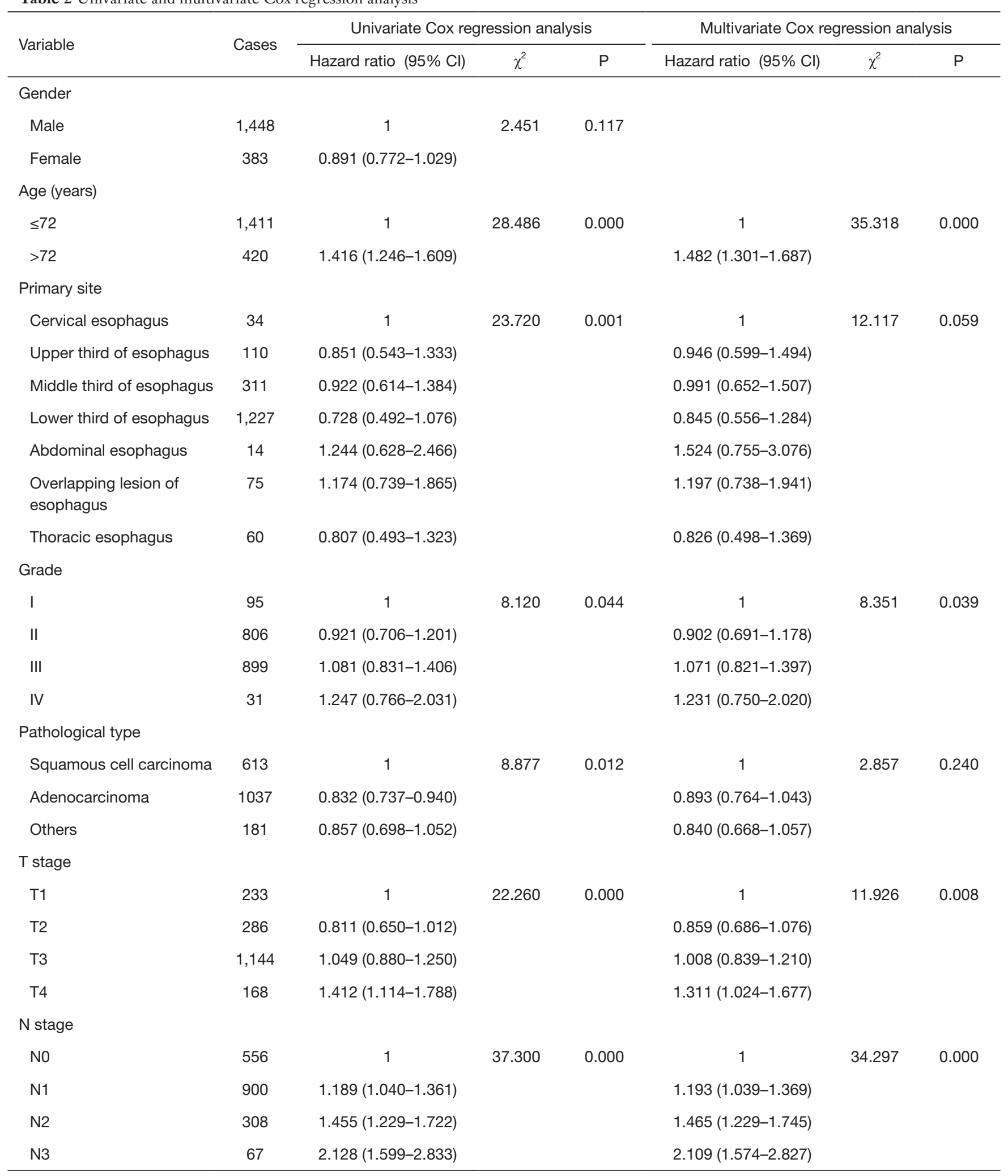

Table 2 (continued) 
Table 2 (continued)

\begin{tabular}{|c|c|c|c|c|c|c|c|}
\hline Variable & Cases & \multicolumn{3}{|c|}{ Univariate Cox regression analysis } & \multicolumn{3}{|c|}{ Multivariate Cox regression analysis } \\
\hline \multicolumn{8}{|c|}{ Tumor size (mm) } \\
\hline$\leq 63$ & 1,325 & 1 & 31.857 & 0.000 & 1 & 17.842 & 0.000 \\
\hline$>63$ & 506 & $1.422(1.259-1.608)$ & & & $1.317(1.159-1.497)$ & & \\
\hline \multicolumn{8}{|l|}{ Race } \\
\hline White & 1,543 & 1 & 7.125 & 0.068 & & & \\
\hline Black & 138 & $1.248(1.020-1.526)$ & & & & & \\
\hline Asian & 132 & $1.195(0.962-1.485)$ & & & & & \\
\hline Others & 18 & $0.815(0.406-1.634)$ & & & & & \\
\hline
\end{tabular}

A

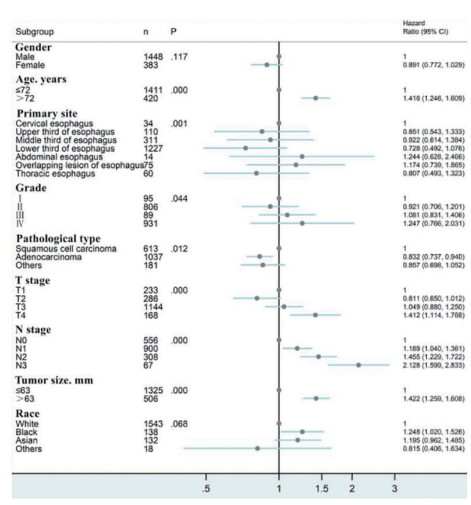

B

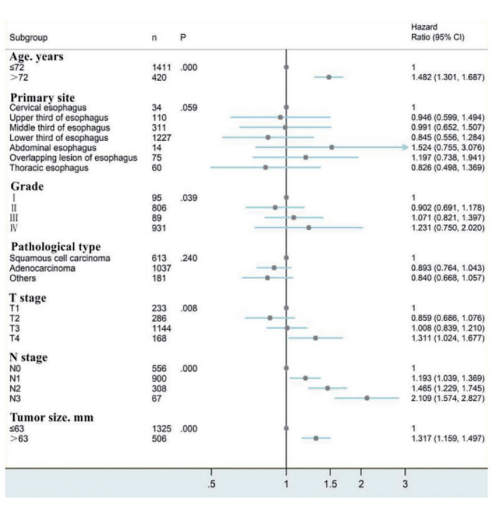

C

Ponits

Grade

T stage

N stage

Age. years

Tumor size. $\mathrm{mm}$

Total points

1-year survival

3-year survival

5-year survival
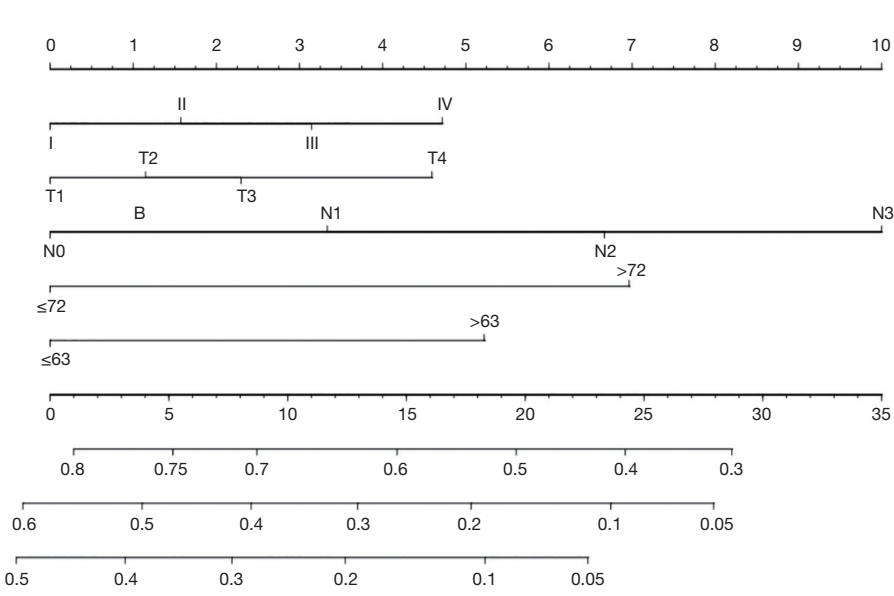

\section{D}

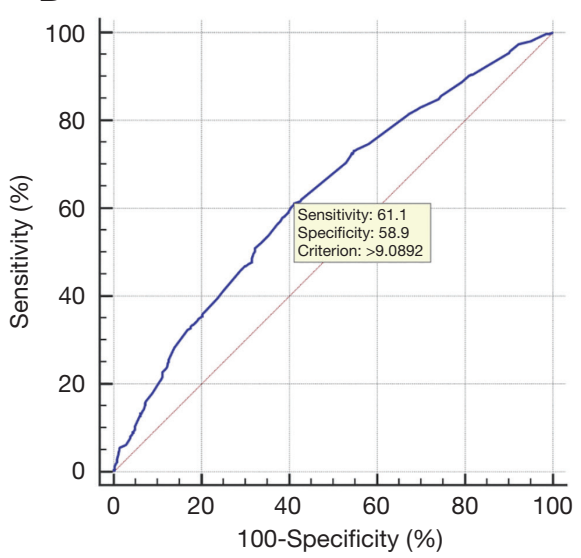

E

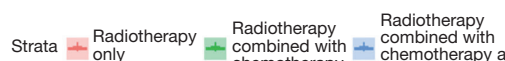

only
chemotherapy $\begin{aligned} & \text { chemotherapy and } \\ & \text { primary site surgery }\end{aligned}$

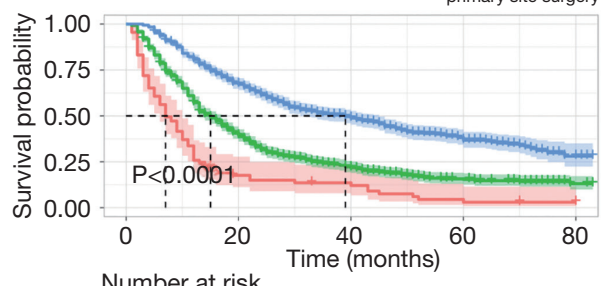

Number at risk
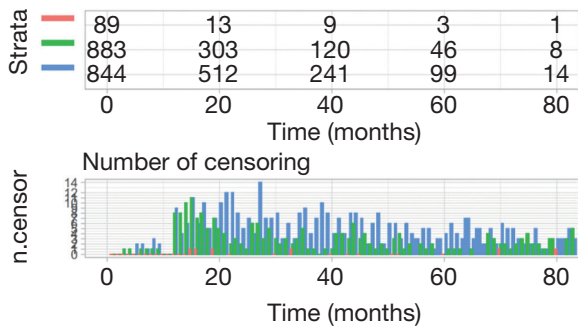

Figure 4 Prognostic analysis of patients without distant metastasis. (A) Forest plots for univariate Cox regression analysis; (B) Forest plots for multivariate Cox regression analysis; (C) construction of the non-distant metastatic prognosis nomogram; (D) validation of the nondistant metastatic prognosis nomogram based on ROC curve; (E) comparison of overall survival among treatment methods. 
Table 3 Comparison of prognoses among different treatment methods

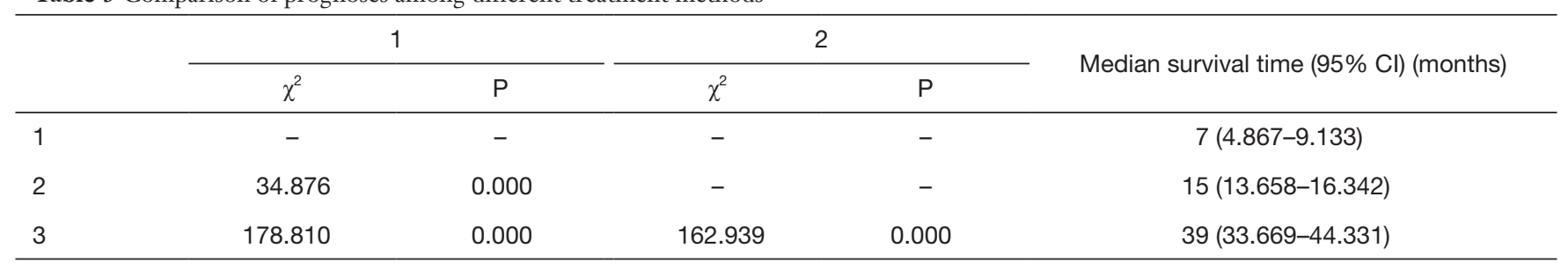

Note: 1 , radiotherapy only; 2 , radiotherapy + chemotherapy; 3 , radiotherapy + chemotherapy + primary site surgery.

Table 4 Univariate and multivariate Cox regression analysis

\begin{tabular}{|c|c|c|c|c|c|c|c|}
\hline Variable & Cases & \multicolumn{3}{|c|}{ Univariate Cox regression analysis } & \multicolumn{3}{|c|}{ Multivariate Cox regression analysis } \\
\hline \multicolumn{8}{|l|}{ Gender } \\
\hline Male & 298 & 1 & 0.256 & 0.613 & & & \\
\hline Female & 52 & $0.922(0.674-1.262)$ & & & & & \\
\hline$\leq 72$ & 277 & 1 & 6.239 & 0.012 & 1 & 4.711 & 0.030 \\
\hline$>72$ & 73 & $1.404(1.076-1.833)$ & & & 1.349 (1.030-1.769) & & \\
\hline \multicolumn{8}{|l|}{ Primary site } \\
\hline Cervical esophagus & 1 & 1 & 1.132 & 0.951 & & & \\
\hline $\begin{array}{l}\text { Overlapping lesion of } \\
\text { esophagus }\end{array}$ & 14 & $1.452(0.188-11.194)$ & & & & & \\
\hline Thoracic esophagus & 17 & $1.088(0.143-8.249)$ & & & & & \\
\hline \multicolumn{8}{|l|}{ Grade } \\
\hline I & 7 & 1 & 8.123 & 0.044 & 1 & 7.258 & 0.064 \\
\hline II & 137 & $0.986(0.460-2.115)$ & & & $1.103(0.510-2.387)$ & & \\
\hline III & 201 & $1.327(0.623-2.826)$ & & & $1.444(0.674-3.093)$ & & \\
\hline IV & 5 & $2.060(0.651-6.519)$ & & & $2.308(0.727-7.332)$ & & \\
\hline $\mathrm{T} 1$ & 97 & 1 & 8.679 & 0.034 & 1 & 8.152 & 0.043 \\
\hline $\mathrm{T} 2$ & 32 & $0.750(0.490-1.148)$ & & & $0.711(0.464-1.090)$ & & \\
\hline T3 & 134 & $0.729(0.553-0.960)$ & & & $0.738(0.559-0.976)$ & & \\
\hline $\mathrm{T} 4$ & 87 & $1.046(0.774-1.414)$ & & & 1.028 (0.757-1.397) & & \\
\hline
\end{tabular}

Table 4 (continued) 
Table 4 (continued)

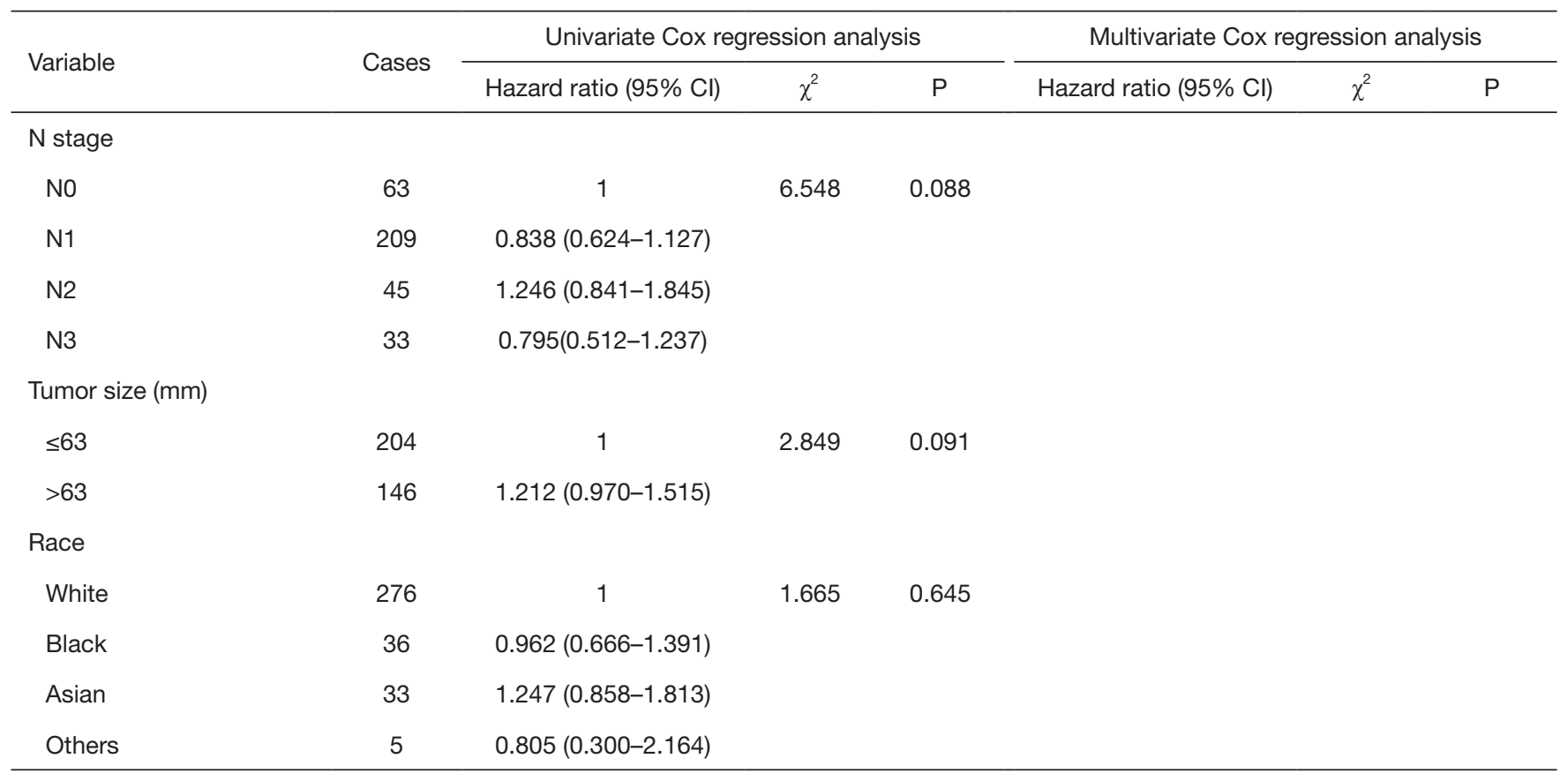
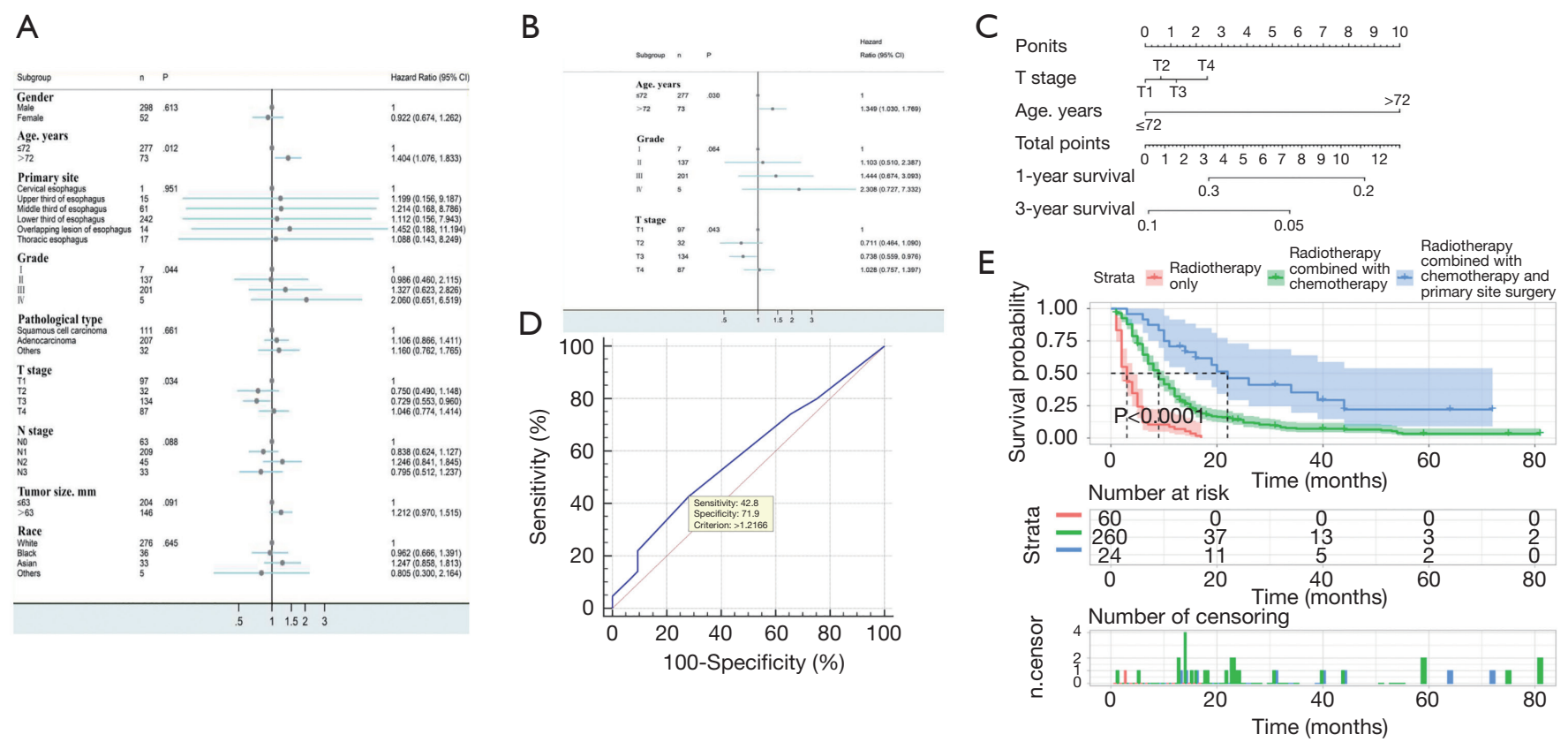

Figure 5 Prognostic analysis of patients with distant metastases. (A) Forest plots for univariate Cox regression analysis; (B) Forest plots for multivariate Cox regression analysis; (C) construction of distant metastatic prognosis nomogram; (D) validation of distant metastatic prognosis nomogram based on the ROC curve; (E) comparison of overall survival among treatment methods. 
Table 5 Comparison of prognoses among different treatment methods

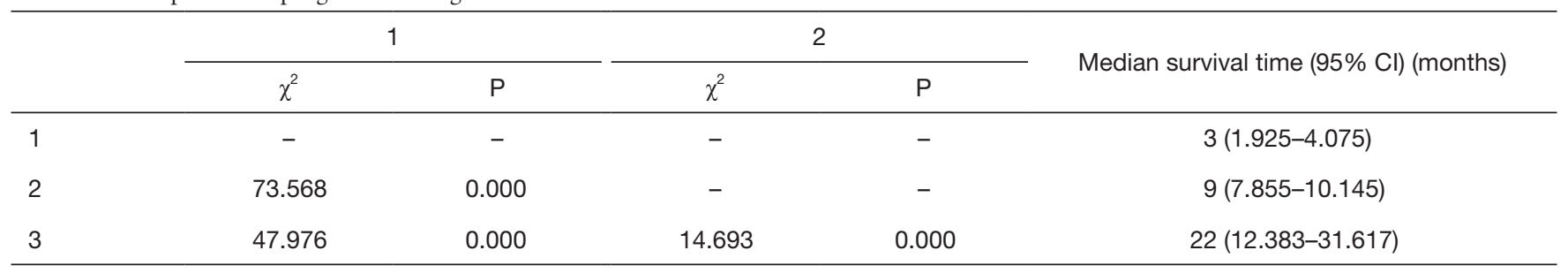

Note: 1 , radiotherapy only; 2 , radiotherapy + chemotherapy; 3 , radiotherapy + chemotherapy + primary site surgery.

used to verify the model, and the AUC was 0.586 (95\% CI: $0.532-0.638 ; \mathrm{z}$ statistic $=1.785 ; \mathrm{P}=0.074)$. In addition, the sensitivity, specificity, and criterion values were 42.8, 71.9, and 1.2166, respectively (Figure 5D).

In all 60 patients received radiotherapy only, 260 patients received radiotherapy + chemotherapy, and 24 patients received radiotherapy + chemotherapy + primary surgery. No analysis was performed for other treatments due to the small number of patients in the other categories. The results showed that the median survival time of patients receiving triple therapy was 22 (95\% CI: 12.383-31.617) months with the best prognosis, while the median survival time of patients receiving radiotherapy only was $3(95 \%$ CI: 1.925-4.075) months with the worst prognosis (Table 5, Figure 5E).

\section{Discussion}

Esophageal cancer is still the sixth leading cause of cancerrelated deaths globally, with more than 500,000 people dying of esophageal cancer each year (1). Although the level of medical diagnosis and treatment continues to rise, due to the lack of clinical symptoms in the early stage of esophageal cancer, most patients are diagnosed at the advanced stage, and prone to distant metastasis, with a median survival time of only about 10 months $(6,7)$. Identifying risk factors that affect the prognosis of patients with esophageal cancer and improving the survival rate of patients are still a clinically urgent problems to be solved $(8,9)$.

To some extent, distant metastases reflect the malignancy and progression of cancer. Patients with distant metastases often enter the terminal stage of the disease, and the prognosis is significantly worse than that of patients without distant metastases. Our study also found that the median survival time for patients with distant metastasis and without distant metastasis was 8.00 (95\% CI: 6.95-9.05) months and 21.00 (95\% CI: 19.55-22.46) months, respectively, with significant differences. For metastatic esophageal cancer, the number of metastatic organs is closely correlated with the progress of the disease. The worse the condition is, the worse the prognosis. It is crucial that clinicians understand these conditions so that the treatment strategy can be chosen rationally. Although positron emission tomographycomputed tomography (PET-CT) and other current examination methods can be used to accurately evaluate the scope of esophageal cancer's whole-body involvement, they are often limited by their high cost and thus do not lend themselves to widespread clinical adoption (10).

The nomogram model is a statistical tool that provides risk prediction for a single patient. It has been used in the assessment of the prognosis of multiple cancers, is considered to have advantages over traditional staging evaluation, and can even serve as a new standard to guide the treatment of cancer patients $(11,12)$. Nomograms are often used not only to predict the survival of patients with all types of cancer but also to successfully quantify risk predictions based on clinicopathological variables (13). This method can provide oncologists with quantification of distant metastasis risk and prognosis in patients with esophageal cancer, thereby helping clinicians to perform more necessary examinations and appropriate treatments, avoid unnecessary aggressive treatments, and ultimately improve patient survival rate and quality of life. This study is the first attempt to use logistic and Cox regression analyses to establish a nomogram prediction model to predict the risk of distant metastasis and prognosis in patients with esophageal cancer. Compared with ROC curve, $\mathrm{X}$-tile considers the time variable when selecting the cutoff point, which is more accurate than ROC curve (14-16). Therefore, using X-tile to determine the optimal critical point can obtain more valuable clinical data. In addition, only one primary tumor of esophageal cancer was selected for this study. The effects of distant metastases from other primary tumors were excluded. The risk of distant metastases from esophageal cancer was only discussed with rigorous scientific statistical methods, which 
makes the results more accurate and reliable and therefore more instructive.

In this study, the purpose of constructing the nomogram prediction model was to predict patients who are prone to distant metastases and to evaluate the prognosis of patients with and without distant metastases. Based on the calculation formula of the prediction model obtained in the nomogram model, we drew the ROC curve to obtain the best cutoff point, so as to judge the level of transfer risk and prognosis. Clinical oncologists can use these results to help reduce the current high mortality rate of esophageal cancer in the future by selecting the most beneficial diagnostic methods for patients. In the logistic regression results of distant metastasis in patients with esophageal cancer, when the total points are greater than 104.8938, the risk of distant metastasis is higher. As evident from the nomogram prediction model, the $\mathrm{N}$ stage and grade contribute the most to the score. The later the $\mathrm{N}$ stage and grade are, the stronger their invasion ability and the greater the risk of distant transfer (17).

Subsequently, independent risk factors for prognosis were analyzed for patients with distant metastases and patients without distant metastases, and nomogram prediction models were drawn. At the same time, the best cutoff point of ROC was used to judge the prognosis. It is evident that age, grade, $\mathrm{T}$ stage, $\mathrm{N}$ stage, and tumor size are independent risk factors that affect the prognosis of patients with no distant metastasis. Looking at the prognostic model of the non-distant metastases nomogram and the cutoff point of prognosis determined by the corresponding ROC curve, we can see that when the total points are greater 9.0892, the prognosis can be considered poor. Age and T stage are independent risk factors that affect the prognosis of patients with distant metastases. Using the prognostic model of the nomogram for distant metastases and the cutoff point of prognosis determined by the corresponding ROC curve, we can see that when the total points are greater than 1.2166, the prognosis can be considered poor. Due to the small number of patients with distant metastases and their short survival time, 5-year cumulative survival cannot be predicted, which has certain implications for the predictive value of the prognostic model. We can see that age and $\mathrm{T}$ stage are important factors influencing the prognosis of patients regardless of whether the patient has distant metastasis. Age can accurately reflect the state and stability of the body, especially for patients with end-stage tumors, and, to a certain extent, reflects the comprehensive physical state of the patient. Several studies have shown that
T stage is closely related to the prognosis of patients with esophageal cancer. T stage is an important indicator of the degree of tumor progression and is often associated with the state of the tumor, which is consistent with the results of this study $(18,19)$. Grade is a manifestation of tumor malignancy and invasiveness. A higher grade often indicates a higher degree of malignancy and the possibility of invasion and metastasis to surrounding tissues and organs, and even to more distant organs. In patients with distant metastases, due to the special characteristics of the included population, grade, $\mathrm{T}$ stage, tumor size, and other factors have not become risk factors affecting prognosis. Of course, due to the short survival of patients with distant metastases, the general risk factors included in the study will also be greatly weakened (20).

In the choice of treatment options, we found that regardless of whether the patient had distant metastases or no-distant metastases, radiotherapy + chemotherapy + primary site surgery was the most effective treatment, followed by combined radiotherapy and chemotherapy. Radiotherapy alone was the least effective treatment. This has certain implications: for patients with good prognosis for esophageal cancer, whether or not distant metastasis occurs, clinical oncologists should consider surgery combined with chemotherapy to improve the prognosis of patients. A randomized trial showed that for patients with locally advanced esophageal cancer, the survival rate of patients with surgery after neoadjuvant chemoradiotherapy was significantly improved, and the pathological response of radiotherapy and chemotherapy was the most critical predictor of survival outcome (21). However, it is still necessary to carry out further prospective research to explore the best candidates for this combination therapy, including factors such as radiation dose, technology, and systemic treatment.

We built a model to assess the risk of distant metastasis in patients with esophageal cancer. A prognostic model for both patients with and without distant metastasis was also constructed. For patients with esophageal cancer, the risk of distant metastasis can be judged first according to the cutoff point of the risk of distant metastasis. For high-risk patients, it is recommended to conduct further imaging examinations of organs to avoid missing distant metastases. Patients can then be classified as distant metastasis or nondistant metastasis according to the examination results, and reasonable treatment recommendations can be given in combination with different prognostic models. If the patient's prognosis is good, radiotherapy + chemotherapy 
+ primary surgery is recommended. If the prognosis is not good, conservative treatment is recommended, with the main treatment purpose being to improve the quality of life. This can effectively balance medical resources and takes into account the burden of patients and families. For example, palliative radiotherapy and chemotherapy can control the growth of EC tumors, improve patients' eating difficulties, maintain body nutrition, improve the quality of life, and also help prolong the survival time of patients. Esophageal stent implantation treatment can provide longlasting esophageal support and expansion, and improve the symptoms of swallowing obstruction caused by malignant esophageal stenosis, thereby alleviating the pain of eating, and improving the nutritional status and quality of life of the patient. and many more.

Naturally, there were limitations to this study which should be mentioned. The related discussion of the patient's treatment plan could not explored more deeply because we do not know what chemotherapy plan the patient received, whether the distant metastases were treated, and what kind of treatment was administered. In addition, the number of cases in the distant metastasis group was too small, which might have had a negative impact on the accuracy of the prognosis prediction. Additionally, the number of cases receiving triple therapy was small, with just 24 cases. A large sample retrospective study is needed to determine whether the patients with distant metastases who received the triple therapy would receive the best treatment results.

In summary, multiple models built in this study yielded prediction formulas, making the model more convenient to use. More importantly, this study offers a comprehensive analysis of the state of esophageal cancer patients, the findings of which can be used to inform rational treatment decisions and improve the survival time and quality of life of patients thereby.

\section{Conclusions}

Our study provided a model for predicting the risk of distant metastasis in patients with esophageal cancer, as well as two personalized models for predicting the prognosis of patients with distant metastasis and patients without distant metastasis. We then visualized the model and provided a simple and convenient calculation formula. In addition, radiotherapy + chemotherapy + primary surgery was determined to be more beneficial to prolonging the survival time of patients with esophageal cancer. However, large sample size and complete clinical information of esophageal cancer are still required for further confirmation of the model.

\section{Acknowledgments}

Funding: This work was supported by Natural Science Basic Research Plan in Shaanxi Province of China (No. 2018JM7090), the Medical Project of Xi'an Science and Technology Bureau [No. 2019114313YX001SF035(1) and 2019114313YX001SF035(6)], and the Scientific Research Plan Projects of the Shaanxi Education Department (19JK0765).

\section{Footnote}

Reporting Checklist: The authors have completed the TRIPOD reporting checklist. Available at https://dx.doi. org/10.21037/jgo-21-429

Conflicts of Interest: All authors have completed the ICMJE uniform disclosure form (available at https://dx.doi. org/10.21037/jgo-21-429). The authors have no conflicts of interest to declare.

Ethical Statement: The authors are accountable for all aspects of the work in ensuring that questions related to the accuracy or integrity of any part of the work are appropriately investigated and resolved. The study was conducted in accordance with the Declaration of Helsinki (as revised in 2013). Institutional ethical approval and informed consent were waived.

Open Access Statement: This is an Open Access article distributed in accordance with the Creative Commons Attribution-NonCommercial-NoDerivs 4.0 International License (CC BY-NC-ND 4.0), which permits the noncommercial replication and distribution of the article with the strict proviso that no changes or edits are made and the original work is properly cited (including links to both the formal publication through the relevant DOI and the license). See: https://creativecommons.org/licenses/by-nc-nd/4.0/.

\section{References}

1. Bray F, Ferlay J, Soerjomataram I, et al. Global cancer statistics 2018: GLOBOCAN estimates of incidence and mortality worldwide for 36 cancers in 185 countries. CA Cancer J Clin 2018;68:394-424. 
2. Shapiro J, van Lanschot JJB, Hulshof MCCM, et al. Neoadjuvant chemoradiotherapy plus surgery versus surgery alone for oesophageal or junctional cancer (CROSS): long-term results of a randomised controlled trial. Lancet Oncol 2015;16:1090-8.

3. Davies AR, Pillai A, Sinha P, et al. Factors associated with early recurrence and death after esophagectomy for cancer. J Surg Oncol 2014;109:459-64.

4. Shaheen NJ, Falk GW, Iyer PG, et al. ACG Clinical Guideline: Diagnosis and Management of Barrett's Esophagus. Am J Gastroenterol 2016;111:30-50; quiz 51.

5. Abraham P, Wang L, Jiang Z, et al. Healthcare utilization and total costs of care among patients with advanced metastatic gastric and esophageal cancer. Future Oncol 2021;17:291-9.

6. Borggreve AS, Kingma BF, Domrachev SA, et al. Surgical treatment of esophageal cancer in the era of multimodality management. Ann N Y Acad Sci 2018;1434:192-209.

7. Nishida N, Yamsaki M, Odagiri K, et al. Combination Therapy With S-1, Oxaliplatin and Leucovorin in Patients With Advanced Esophageal Squamous Cell Carcinoma. In Vivo 2019;33:2249-54.

8. Wu N, Pang LW, Chen ZM, et al. Tumour length is an independent prognostic factor of esophageal squamous cell carcinomas. Chin Med J (Engl) 2012;125:4445-8.

9. Matsumoto Y, Kimura K, Zhou Q, et al. Treatments and outcomes of older patients with esophageal cancer: Comparison with younger patients. Mol Clin Oncol 2019;11:383-9.

10. Wong R, Walker-Dilks C, Raifu A. Evidence-based guideline recommendations on the use of positron emission tomography imaging in oesophageal cancer. Clin Oncol (R Coll Radiol) 2012;24:86-104.

11. Karakiewicz PI, Briganti A, Chun FK, et al. Multiinstitutional validation of a new renal cancer-specific survival nomogram. J Clin Oncol 2007;25:1316-22.

Cite this article as: Zhang M, Cui M, Zuo Q, Wang L, Wang J, Zhu L, Yan R, Lu N, Yan H, Zhang L. Construction and evaluation of prognostic models for esophageal cancer patients with distant and non-distant metastases: providing a reference process for clinical diagnosis and treatment. J Gastrointest Oncol 2021;12(4):1241-1254. doi: 10.21037/jgo-21-429
12. Wang Y, Li J, Xia Y, et al. Prognostic nomogram for intrahepatic cholangiocarcinoma after partial hepatectomy. J Clin Oncol 2013;31:1188-95.

13. Wang $M$, Gao $Y$, Feng $H$, et al. A nomogram incorporating six easily obtained parameters to discriminate intrahepatic cholangiocarcinoma and hepatocellular carcinoma. Cancer Med 2018;7:646-54.

14. Camp RL, Dolled-Filhart M, Rimm DL. X-tile: a new bio-informatics tool for biomarker assessment and outcome-based cut-point optimization. Clin Cancer Res 2004;10:7252-9.

15. Sun $\mathrm{H}, \mathrm{He} B, \mathrm{Nie} Z$, et al. A nomogram based on serum bilirubin and albumin levels predicts survival in gastric cancer patients. Oncotarget 2017;8:41305-18.

16. Li X, $\mathrm{Lu} \mathrm{H}, \mathrm{Xu} \mathrm{K}$, et al. Negative lymph node count is an independent prognostic factor for patients with rectal cancer who received preoperative radiotherapy. BMC Cancer 2017;17:227.

17. Ai D, Zhu H, Ren W, et al. Patterns of distant organ metastases in esophageal cancer: a population-based study. J Thorac Dis 2017;9:3023-30.

18. Arigami T, Uchikado Y, Omoto I, et al. Primary Tumor Score Based on Tumor Depth and Length Predicts Prognosis in Esophageal Squamous Cell Carcinoma. Anticancer Res 2018;38:5447-52.

19. Wang H, Deng F, Liu Q, et al. Prognostic significance of lymph node metastasis in esophageal squamous cell carcinoma. Pathol Res Pract 2017;213:842-7.

20. Liu J, Wei Z, Zhang J, et al. Which factors are associated with extremely short-term survival after surgery in patients with esophageal squamous cell carcinoma? Asia Pac J Clin Oncol 2016;12:308-13.

21. Huang FL, Yu SJ. Esophageal cancer: Risk factors, genetic association, and treatment. Asian J Surg 2018;41:210-5.

(English Language Editors: G. Stone and J. Gray) 